\title{
Office/home-based exercise rehabilitation is useful for computer-based workers in today's world
}

\author{
Ardalan Shariat ibttps://orcid.org/0000-0003-4750-5797
}

It can be said that in today's world, any person from a 10-yearold child to a 70-year-old man or woman uses a smartphone or laptop or PC for different purposes such as communication, entertainment, doing business, and other daily duties. The lifestyle predominates not only in developed countries but also in developing countries where individuals spend so much time (around $8 \mathrm{hr} /$ day) for sitting on their chair. Office workers will often not have enough time for participation in physical activities. In addition to decrease in the range of motion, muscles slowly get weak and can atrophy especially the multifidus muscles. Chronic pains in neck, shoulder, and lower back can lead to insomnia. Following insomnia, cognitive performance, physiological performance and mood will be affected as well.

Exercise rehabilitation is an established therapy in developed countries and in recent decades it is slowly getting known in developing countries. Exercise is a nonpharmacological treatment for individuals who suffer from physical, physiological or psychological issues. Exercise rehabilitation is not only effective on physical issues but also can improve physiological and psychological aspects of individuals in the workplace and home.

Unfortunately, most of the prescriptions have focused on exercise rehabilitation for specific diseases undertaken under supervi- sion of a clinic-based therapist with specific equipment. There is a lack of knowledge about different standard packages of feasible exercise rehabilitation for individuals of different gender in different ages who do not have a time to go to gym or visit a therapist. The home or office-based packages should be easy to do without need for specialist equipment or instruments and come with clear instructions.

The Editorial Board of the Journal of Exercise Rehabilitation invites scientists and therapists to submit the results of their studies with critical thinking, and introduces their suggested exercise rehabilitation protocols for computer-based workers with an international perspective.

\section{CONFLICT OF INTEREST}

No potential conflict of interest relevant to this article was reported.

Editor of the Journal of Exercise Rehabilitation Sports Medicine Research Center, Neuroscience Institute, Tehran University of Medical Sciences, Tehran, Iran E-mail: a-shariat@razi.tums.ac.ir 